Stärkung der Allgemeinmedizin

\section{Reform des}

\section{Medizinstudiums kommt}

Die Bundesregierung und die Gesundheits- und Wissenschaftsminister der Länder haben Ende März den „Masterplan Medizinstudium 2020" beschlossen. Darin einigten sich Bund und Länder auf eine Veränderung der Studienstruktur und der Ausbildungsinhalte.

Wie das Bundesministerium für Gesundheit (BMG) bekanntgab, sollen die angehenden Mediziner neben dem bisherigen Ausbildungsschwerpunkt hoch spezialisierter Fälle an Universitätskliniken künftig auch alltägliche Erkrankungen in der ambulanten und stationären Praxis kennenlernen. Dies soll im Praktischen Jahr durch ein Quartal in der ambulanten Versorgung ermöglicht werden. Darüber hinaus sieht der Masterplan vor, die Allgemeinmedizin zu stärken.

Auch die im Vorfeld häufig diskutierte Landarztquote findet sich in der Reform wieder. Künftig sollen die Länder bis zu zehn Prozent der Medizinstudienplätze an Bewerber vergeben, die sich dazu verpflichten, nach Studienabschluss bis zu zehn Jahre lang als Hausarzt in unterversorgten Gebieten zu arbeiten.

„Der Masterplan Medizinstudium 2020 ist ein wichtiger Schritt hin zu einem modernen Medizinstudium, das unsere Ärztinnen und Ärzte auf die künftigen Herausforderungen vorbereitet und eine gute Patientenversorgung überall in Deutschland auch in Zukunft sicherstellt. Mehr Praxisbezug im Studium und eine Stärkung der Allgemeinmedizin sind gerade mit Blick auf die gute Versorgung im ländlichen Raum von großer Bedeutung", erklärte Bundesgesundheitsminister Hermann Gröhe (CDU) bei der Vorstellung des Reformpakets.

cas

\section{KZBV und Kassen einigen sich auf Grundsatzfinanzierung}

Kassenzahnärztliche Bundesvereinigung (KZBV) und GKVSpitzenverband haben sich Mitte April auf eine Grundsatzvereinbarung für die Finanzierung der ersten Stufe des OnlineRollouts in Zahnarztpraxen geeinigt. Wie die KZBV mitteilte, werden die Kassen die Ausstattungs- und Betriebskosten für die Anbindung der Praxen an die Telematikinfrastruktur (TI) vollständig übernehmen. Die Kostenübernahme erfolge auf Grundlage von Erstattungs- und Betriebskostenpauschalen. Die konkrete Höhe der Pauschalen wird der KZBV zufolge in einer weiteren Vereinbarung festgelegt. KZBV und GKV-Spitzenverband einigten sich darauf, dass die Pauschalen ein Standard-Erstausstattungspaket und ein Standard-Betriebspaket vollständig abdecken werden. Zahnärzte würden somit eine vollumfängliche Rückerstattung erhalten, wenn sie die günstigsten Komponenten und Dienste bestellen.

„Damit ist für die Praxen eine vollständige Finanzierung sowohl der Erstausstattung als auch der Betriebskosten gewährleistet. Eine vollständige Finanzierungsvereinbarung zu schließen, wenn über Preise und konkrete Abwicklung noch nichts bekannt ist, war für beide Seiten allerdings eine ganz besondere Herausforderung", hieß es in einem Pressestatement der KZBV.

cas

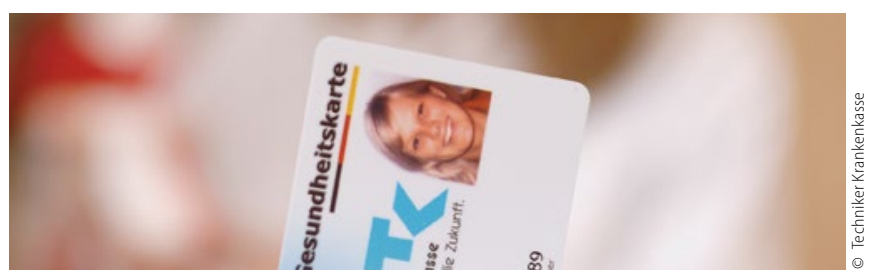

\title{
Deutsche und Gesundheit: Bitte mehr staatliche Kontrolle!
}

Mehr als die Hälfte der Deutschen wünscht sich einen stärkeren Staat, zumindest was die Gesundheit der Bevölkerung angeht: Mehr als 1.000 Teilnehmer gaben in einer Umfrage des Bundesinstituts für Risikobewertung (BfR) für den Verbrauchermonitor 2017 an, dass es dafür mehr Verbote und Beschränkungen von staatlicher Seite geben dürfe.

Ein Drittel der Befragten wünscht sich mehr wissenschaftlich gesicherte Informationen vom Staat, mit deren Hilfe jeder eigen-

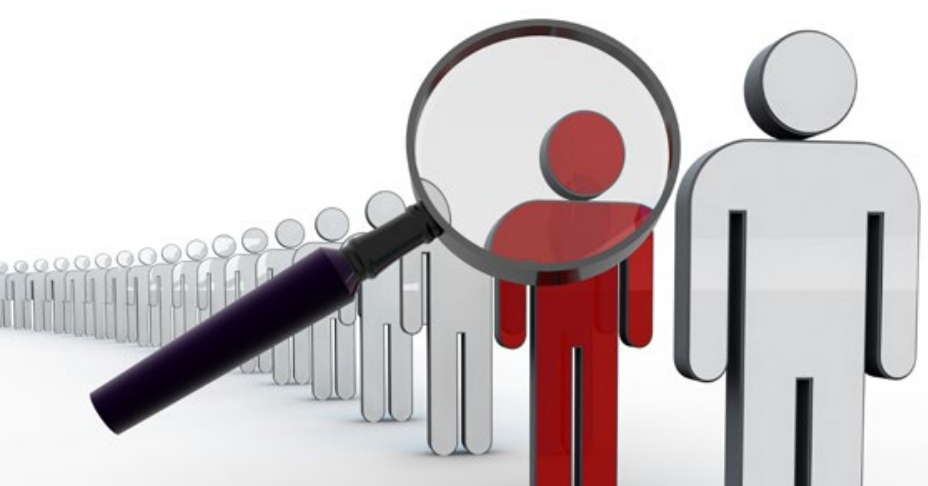

verantwortlich gesundheitliche Risiken beurteilen könne. Letztere selbst abzuschätzen, dazu fühlt sich nur etwa jeder achte Bundesbürger in der Lage.

Als eine der größten Gesundheitsgefahren bewerteten 19 Prozent der Befragten das Rauchen, gefolgt von Klima- und Umweltbelastungen (17 Prozent). Auf dem dritten Platz liegen ungesunde Ernährung und Alkoholkonsum gleichauf (jeweils 14 Prozent).

Was die Lebensmittelsicherheit angeht, empfinden die Deutschen vor allem Antibiotika-Resistenzen als beunruhigend (59 Prozent), doch auch gentechnisch veränderte Lebensmittel (56 Prozent) sowie Reste von Pflanzenschutzmitteln in Lebensmitteln (52 Prozent).

In halbjährlichem Abstand liefert der BfR-Verbrauchermonitor als repräsentative Bevölkerungsbefragung Antworten auf die Frage, wie die Öffentlichkeit zu Themen aus dem Bereich des gesundheitlichen Verbraucherschutzes steht. 